\title{
Sentencia judicial, delito sexual y pericial psicológica: enfoque transcultural ${ }^{*}$
}

\section{Judicial Sentence, Sexual Assaults and Psychological Expertise: A Cross-Cultural Approach}

Recepción: 22 Junio 2015 | Aprobación: 01 Diciembre 2017

\author{
Milena Martínez Rudas ${ }^{\mathrm{a}}$ \\ Universidad de la Costa CUC, Colombia \\ ORCID: http://orcid.org/0000-0001-5450-8932 \\ Stefany Baena Valencia \\ Universidad de la Costa CUC, Colombia \\ TITO José CRISSIEN \\ Universidad de la Costa CUC, Colombia \\ Ismael Pérez García \\ Universidad de Santiago de Compostela, España \\ Jesús Santolaya Prego de Oliver \\ Universidad Internacional de Valencia, España
}

\footnotetext{
a Autor de correspondencia. Correo electrónico: mmartine49@cuc.edu.co
}

Para citar este artículo: Martínez Rudas, M., Baena Valencia, S., Crissien, T. J., Pérez García, I., \& Santolaya Prego de Oliver, J. (2018). Sentencia judicial, delito sexual y pericial psicológica: enfoque transcultural. Universitas Psychologica, 17(2),

1-11. https://doi.org/10.11144/Javeriana.upsy17-2.sjds

\section{RESUMEN}

Se analiza la influencia de la pericial psicológica en las decisiones judiciales en delitos sexuales infantiles en tres departamentos de Colombia y una provincia española. El estudio transcultural compara las sentencias entre los años 2000 y 2014. Los resultados arrojaron que en el 91.7 $\%$ de sentencias de juicios celebrados, los magistrados españoles hacen referencia explícita a la pericial psicológica. En tanto que en Colombia, la proporción fue del $88.8 \%$. La cercanía de estos porcentajes es alentadora, porque a estos resultados se llega utilizando examen de datos distintos, desiguales códigos penales y recorridos dispares en la aplicación de la pericial psicológica. Se obtienen conclusiones similares que refrendan la importancia del psicólogo en la toma de decisiones judiciales.

Palabras clave

pericial psicológica; sentencia judicial; abuso sexual infantil; informe psicológico forense; jueces.

\section{ABSTRACT}

It was analyze the influence of psychological evaluation in judicial decisions of child sexual abuse in three departments of Colombia and in a Spanish province. The transcultural analysis compares judgments of child sexual abuse in both countries between the year of 2000 and 2014. The $91.7 \%$ of judgments of the Spanish judges or magistrates made explicit reference to the psychological evaluation while in Colombia the proportion was $88.8 \%$. The proximity of these percentages is encouraging because these results were reached using different test data, different criminal codes and different routes in the application of psychological expertise. Similar conclusions in both countries confirm the value of the contribution of psychologist on judicial decisions.

Keywords 
psychological expertise; judicial sentence; child sexual abuse; psychological report; judges.

La violencia sexual en un problema universal. Los resultados de metaanálisis confirman que el abuso sexual infantil es un problema global (Stoltenborgh, Van Ljzendoorm, Eurser, \& Bakermans-Kranenburg, 2011). Constituye un fenómeno complejo, resultante de una combinación de factores individuales, familiares y sociales. La gravedad de esta conducta ha sido elevada transculturamente a la categoría de delito. Esto quiere decir que representa una conducta punible legítimamente por los mecanismos de regulación social (Hernández, 2008 citado en Tapias, 2011). En los delitos sexuales, al no existir testigos directos del hecho, la prueba pericial resulta uno de los elementos más valiosos en el proceso de búsqueda de justicia (Pompeyo \& López, 2014, p.10). En este contexto, la psicología forense se entiende como la rama de la psicología jurídica que se encarga de brindar el soporte técnico a la ley, a través de conceptos que son solicitados por alguna autoridad judicial (Soria \& Saiz, 2006). De ahí, la frecuencia de las actuaciones del profesional en la realización de exámenes psicológicos de niños en los casos de delitos sexuales. La psicología forense debe aportar elementos básicos de la pericia psicológica tales como la narración de la víctima por medio de protocolos de entrevista especializados y el análisis de esta a través de técnicas de credibilidad de testimonio, así como la evaluación del daño psicológico con el fin de poder presentar un informe idóneo, aportante a la administración de Justicia (Tapias, 2011).

El informe psicológico es frecuentemente la única forma de comunicación de los resultados de la evaluación; es un documento que demuestra que se ha llevado a cabo una evaluación y finalizado la producción de inferencias (Simoes, 2001). En este sentido, en los procesos legales, el psicólogo es un perito designado por el juez o por las partes y, como especialista, presenta por escrito $\mathrm{u}$ oralmente sus opiniones. A veces, esta es toda su intervención. En otras ocasiones, se le puede solicitar que explique pormenorizadamente ante el tribunal cómo llegó a las conclusiones presentadas en el informe.

En Colombia, al psicólogo se le considera un perito en la medida en que posee experiencia y conocimientos especializados de naturaleza técnica, científica y práctica (artículo 242, Ley 600 del 2000) que escapan a la cultura general de la persona. Asimismo, peritaje o dictamen es el mismo informe psicológico; se conoce también como peritazgo o experticio (Ley 906 del 2004), y consiste en un proceso de evaluación que se presenta ante la autoridad competente.

La misma Ley 600 del 2000 o Código de Procedimiento Penal Colombiano, en su Título VI, consigna todo lo concerniente a la prueba. Es donde se amplían las funciones del perito. El Instituto Nacional de Medicina Legal y Ciencias Forenses es el principal referente de los servicios de peritaje, en particular de los delitos contra la vida e integridad personal o los denominados contra la libertad y el pudor sexual. Haciendo un análisis de la Ley 600, se evidencia que durante los años de vigencia, 2000-2004, esta amparaba las actuaciones procedimentales en materia judicial e incluía la prueba pericial dentro de sus procedimientos, sin especificar el caso de los informes periciales psicológicos dentro del marco de los delitos sexuales.

El Instituto Nacional de Medicina Legal y Ciencias Forenses de Colombia es la entidad de mayor preponderancia en lo referente a los dictámenes sexológicos médicos; inicialmente, los psicológicos y psiquiátricos no tenían un papel trascendental dentro de los diferentes procesos. Posteriormente, con las modificaciones de la Ley 600 de 2000 en la Ley 906 de 2004, se expide el Nuevo Código de Procedimiento Penal, observando así un gran cambio en la normatividad y en el papel de los peritos, el cual se hace más activo, dinámico y relevante dentro de la actuación procesal, superando la pasividad de la ley anterior.

En Colombia, el delito sexual es entendido como cualquier actividad sexual entre dos personas sin consentimiento de una. Puede producirse entre adultos, de un adulto a un menor o incluso entre menores; está constituido por actos, o amenazas, que atentan contra la 
integridad y la formación sexual de una persona y puede ser ejercido mediante la fuerza física o psicológica o en ausencia total del uso de la fuerza, con el propósito de imponer una conducta sexual en contra de la voluntad.

Existen dos tipos de abuso sexual. Uno, con contacto, en el cual están las acciones de frotamiento, besos, caricias, penetración digital en recto, penetración digital en vagina, penetración vaginal $\mathrm{u}$ oral, entre otras. Y otro, sin contacto, que está relacionado con comportamientos tales como exhibicionismo, desnudez, fotografías de carácter sexual, ser testigos de actos sexuales con terceros, entre otras. La gran variedad de definiciones de abuso sexual en niños ha causado muchos problemas de interpretación de los hallazgos. Tanto las definiciones legales como médicas son restrictivas y se suelen apoyar en la evidencia física del abuso, que normalmente no está presente (Mrazek, 1983). La dificultad radica en que los hallazgos periciales en niños y niñas que han sido víctimas pueden ir desde serias lesiones físicas, hasta la ausencia total de evidencia objetiva de la agresión (Rodríguez, 2010).

En materia penal, el proceso para las investigaciones en delitos sexuales, según la Ley 600 de 2000, se desarrollaba a través de las etapas de investigación previa, instrucción y juzgamiento, sin dar lugar a la sustentación del informe o peritaje psicológico. Así permaneció la situación hasta el 2005, cuando en Colombia, inicialmente en el Eje Cafetero y Bogotá, se cambió a un Sistema Penal Oral Acusatorio, y este constituye precisamente una de las razones por las que el estudio transnacional con la muestra colombiana que se ofrece a continuación abarca el cuatrienio 2000-2004, tiempo al que se circunscribe la vigencia de la Ley 600 del 2000. Pero, dado que los informes psicológicos son consultivos, lo importante de este estudio es la consideración que el juez le otorgará al informe.

En España, la progresión epidemiológica del delito sexual en la infancia y adolescencia es clara. El Centro Reina Sofía realizó, en el año 2004, un estudio sobre los expedientes a menores incoados por los Servicios Sociales entre 1997 y 1998 (cerca de 33 000). Esta investigación, titulada "Maltrato infantil en la familia, España (1997/1998)", confirmó la existencia de 11148 menores víctimas de maltrato en el ámbito familiar. El $86.37 \%$ de estos menores sufría negligencia, el $35.38 \%$ maltrato psicológico, el $19.91 \%$ maltrato físico y solo el 3.55\% abuso sexual (Centro Reina Sofía para el Estudio de la Violencia, 2004).

Años más tarde, la Fundación Ayuda a Niños y Adolescentes en Riesgo ([Anar], 2012), publica, con motivo del Día Internacional de la Lucha contra el Maltrato Infantil, un informe en el que reveló que durante el año 2012 atendió los casos de 1778 niños y adolescentes víctimas de violencia, en su mayoría por maltrato físico $(28.7 \%)$, pero también por abuso sexual $(11.5 \%)$ y por pornografía infantil (0.8\%). El Consejo de Europa, consciente de la extensión de la victimización sexual contra menores en los distintos países europeos, ha impulsado una campaña de sensibilización titulada "One in five" (Uno de cada cinco), que resume los porcentajes obtenidos en los estudios retrospectivos de prevalencia en delitos sexuales (Pereda, 2016).

El estudio transcultural de la investigación que nos ocupa presenta delitos de índole sexual infligidos a niños y niñas o adultos discapacitados. Se trata de hechos que atentan contra la libertad de elección sexual de la persona, o que promueven la sexualidad cuando el sujeto pasivo (víctima) es menor de la edad de consentimiento estipulada por la ley o incapaz.

Estas transgresiones están reguladas en el Título VIII del Código Penal Español, enmarcadas en los "Delitos contra la libertad e indemnidad sexuales". La indemnidad sexual se relaciona directamente con la necesidad de proteger y garantizar el desarrollo normal en el ámbito sexual de quienes aún no han alcanzado el grado de madurez suficiente, como es el caso de las y los menores y, asimismo, de quienes por anomalías psíquicas, carecen de plena capacidad para llegar a tomar conciencia de lo que significa una relación sexual. Entre los delitos regulados están los abusos y agresiones sexuales a menores, los delitos de exhibicionismo y provocación 
sexual y los delitos de prostitución y la corrupción de menores.

La diferencia básica estriba en que en el supuesto de la agresión sexual se exige que aquel que atenta contra la libertad sexual de la víctima lo haga con violencia o intimidación. Mientras que en el caso del abuso sexual, la conducta punible se realiza sin violencia e intimidación, e igualmente sin que medie consentimiento o capacidad para otorgarlo. En ambos supuestos existen tipos agravados que, según determinadas circunstancias, elevan la pena al agresor.

Ahora bien, el abuso sexual debe ser definido desde dos grandes conceptos: el de coerción y el de asimetría de edad. La coerción denota presión, fuerza física y engaño; la asimetría de edad refiere a que la víctima no está siendo verdaderamente libre de decisión, por lo cual esto no representa una actividad sexual común, ya que los que hacen parte (víctima y agresor) tienen un grado de madurez biológica, experiencias y expectativas muy diferentes.

En España, el punto de confluencia entre el derecho y la psicología lo ratifica el artículo 10 de la Constitución de 1978. Ocho años más tarde, en 1986, la Dirección General de Relaciones con la Administración de Justicia contrata los primeros psicólogos. Ese respaldo laboral convierte al psicólogo forense en un experto que asesora al juez y al resto de operadores jurídicos, aunque su dictamen como perito es valorado o criticado según el interés de las partes (modelo del adversario). Pero, cuando un juez llega a conclusiones diferentes a las de los informes, debe expresar las razones que justifiquen tal decisión (Sentencia del Tribunal Supremo, de 6 de marzo de 1995). Pese a que para satisfacer los requisitos de la presunción de inocencia de un acusado, reiteradas jurisprudencias del Tribunal Supremo exigen el cumplimiento de tres premisas: ausencia de incredibilidad subjetiva (descarta el móvil del resentimiento, la enemistad y/o la venganza en la denuncia), persistencia en la incriminación (esta debe prolongarse en el tiempo sin ambigüedades ni contradicciones) y verosimilitud del testimonio (constatación objetiva de la existencia del hecho). En la observancia de esta última condición, el psicólogo forense tiene un papel determinante para verificar secuelas, credibilidad del testimonio de las víctimas o ambas huellas. Porque resulta habitual que los tribunales soliciten examen psicológico de aquellos menores que denuncian abusos sexuales (Tejero \& González, 2009). El derecho recurre a la psicología para la producción de la carga de la prueba y para el cuidado de las víctimas (Granjeiro \& Costa, 2010). Para Raskin y Steller (1989), la contribución de la psicología se ocupa del estudio de la credibilidad del testimonio en menores y/o discapacitados, víctimas de delitos contra la libertad sexual (abuso, agresión y mixto), mediante el Análisis de Contenidos Basado en Criterios (CBCA) y, como elemento central, la Evaluación de la Validez de las Declaraciones a través de la SVA (Statement Validity Assessment).

Las víctimas de delitos sexuales pueden presentar lesiones y evidencias físicas, pero en muchos sucesos de esta índole las señales no están presentes, caso en el cual es indispensable el peritaje psicológico forense. Los casos denunciados por violencia sexual justifican por sí mismos el nombramiento judicial del psicólogo forense para efectuar valoraciones en la instrucción penal. Desde diferentes perspectivas, diversos estudios han sido diseñados para explicar el porqué de las decisiones judiciales y los factores que influyen en las sentencias. "La vida de la ley no es lógica sino experiencia", ya que emitir repetidas resoluciones puede aumentar la probabilidad de que los jueces, para simplificar sus decisiones, acepten recomendaciones exógenas (Aliste Santos, 2011).

López y Pueyo (2007) concluyen que cuando se analiza la relación entre los peritajes psicológicos y las sentencias, se observa que en el $88 \%$ de los casos, los jueces y los psicólogos llegan a conclusiones similares. Moreno, Correa y Pérez (2013) hallaron que el $73 \%$ de las veces en las que se cita la pericial psicológica en la sentencia judicial, esta va aparejada con abuso sexual. Rudas, Baena y Pérez (2016) reportan que en un $80 \%$ de los casos, el juez tomó su decisión de manera concordante con 
los resultados del informe psicológico forense. Para constatar la validez intercultural, Collings, Lindblom, Madu y Park (2009) examinan la validez transcultural del abuso sexual infantil en muestras de adolescentes y adultos jóvenes de tres países: Sudáfrica $(\mathrm{n}=200)$, Corea $(\mathrm{n}=200)$ y Suecia $(n=200)$. Los participantes completan la Escala de Mito del Abuso Sexual Infantil (CSAMS) de 15 ítems. Para cada país, los datos se analizan mediante análisis de factores del eje principal con rotación Varimax. Las respuestas sobre el CSAMS obtenido para la muestra de validación original de Sudáfrica se replican en las muestras sueca y coreana; se observa una estructura psicológicamente significativa.

El presente trabajo es el hilo conductor del contexto hispanocolombiano de abuso sexual infantil unido a la judicatura y objeto de estudio. El propósito de esta revisión comparativa es determinar la influencia de los informes o pericias psicológicas forenses, en las decisiones judiciales en un contexto transcultural: Colombia y España. Es decir, iqué uso hace de la prueba pericial el juzgador de delitos sexuales infantiles (o discapacitados) en España y en Colombia? ¿Para el buen funcionamiento de la Justicia, el derecho puede resolver las situaciones de abuso sexual solo con códigos y leyes?

\section{Método}

\section{Muestra}

Se llevó a cabo el análisis de 72 sentencias de delitos sexuales emitidas en segunda instancia por los Tribunales Superiores en Colombia; concretamente, en los departamentos de Cauca, Nariño y Caldas, suponen el 100 \% (la totalidad) de las sentencias emitidas por abuso sexual infantil en el periodo analizado que coincide con la aplicación de la reforma del Código Procedimiento Penal (cambio de la Ley 600 de 2000 a la Ley 906 de 2004). Por otro lado, se contó con 85 sentencias judiciales de delitos sexuales infantiles, con sus correspondientes informes psicológicos forenses, en vistas celebradas en Santa Cruz de Tenerife,
España, en juzgados penales $(65.5 \%)$ y secciones de la audiencia (34.5\%). El criterio de selección fue de muestreo probabilístico simple sin reemplazo, de una muestra total $(\mathrm{N})$ de 300 vistas orales practicadas entre 2008 y 2014, con un error estándar $<0.01$, la muestra $\mathrm{n}$ es $=74.4(\mathrm{n}$ $\left.=\mathrm{n}^{\prime} / 1+\mathrm{n}^{\prime} / \mathrm{N}\right)$, se utilizan 85 . La muestra total es de 157 sentencias.

\section{Procedimiento}

Esta investigación concluye acerca de si se tiene en cuenta o no el peritaje psicológico en las decisiones judiciales en los departamentos de Nariño, Cauca y Caldas en Colombia entre los años 2000 a 2004. Por otro lado, se comparó con sentencias judiciales emitidas en la provincia de Santa Cruz de Tenerife, España, entre los años 2008 a 2014, por denuncias de naturaleza sexual a menores de edad $(87.5 \%$ representado así: $61.1 \%$ de género femenino, edad media de 8.1 años, desviación estándar 2.8 y moda 9.2 años y $38.9 \%$ de género masculino, edad media de 8.5 años, desviación estándar 1.9 y moda 9.6 años) o mujeres adultas discapacitadas (12.5\%, con edad mental equivalente media de 8.6 años, desviación estándar 3.1 y moda 9 años de edad equivalente). Se intentó esclarecer en qué medida los jueces estiman las conclusiones de informes psicológicos forenses que difieren, para argumentar la responsabilidad, sobreseimiento o absolución de los acusados más allá de los periodos temporales de obtención de las muestras. Aunque las muestras sí están unidas por el delito sexual cometido a menores y la convergencia entre el derecho y la psicología para garantizar el mejor funcionamiento de la Justicia, que son heurísticos universales.

\section{Instrumentos de medida}

Para estructurar la información recogida y para articular las dos muestras (colombiana y española), se diseñaron dos protocolos: (a) uno dicotómico con recogida de sentencias judiciales, que incluye la opción de que el juez o magistrado haga alusión explícita al 
informe pericial psicológico forense en el texto de la sentencia o no aluda a él (presencia/ ausencia) y (b) otro protocolo que valora la influencia del informe psicológico en la decisión judicial (por ejemplo, si el fallo judicial concuerda o no con la pericial, aunque no haya referencia tácita escrita a esta en el texto de la sentencia). Existe otro protocolo para los informes periciales que contiene las variables comunes a las dos muestras: huella de memoria (credibilidad o no credibilidad del testimonio, determinada a partir de la aplicación de la SVA para la muestra española; mientras que para la muestra colombiana se tiene en cuenta la conclusión emitida por el perito) y huella psíquica (verificación de secuelas psicológicas primarias en las dos muestras). Como variable dependiente (VD) de este estudio se utilizó la mención del informe pericial en las 157 sentencias revisadas.

Por otro lado, para homogenizar los delitos sexuales infantiles españoles y colombianos salvando los matices de los códigos penales nacionales, se crearon tres subvariables: a) violencia: hace referencia a cualquier tipo de contacto físico sin consentimiento, con o sin acceso carnal, pero con violencia o intimidación; b) sexual: conlleva contacto físico, que puede incluir penetración vaginal, anal, oral, digital, caricias o proposiciones verbales explícitas, hechas sin violencia o intimidación, pero sin consentimiento de la víctima y c) mixta: aglutina otros tipos de delitos sexuales infantiles sin contacto físico (p. ej., exhibicionismo y pornografía). Los datos obtenidos se analizan estadísticamente a través del paquete estadístico SPSS 21 de modo correlacional chi-cuadrado.

\section{Resultados}

Se encontró que en Colombia, de los casos analizados en el periodo 2000-2004, el 63.8\% (46) corresponde al delito de violencia y el $36.1 \%$ restante pertenece al delito de tipo sexual, con 26 casos; se documentan 11 casos de tipo mixto. En Santa cruz de Tenerife, se obtiene que de las 85 sentencias emitidas durante los años 2008 a 2014, el 40\% (34) concierne a los casos de violencia y el $51.8 \%$ de los casos (44) corresponde al tipo sexual, los siete casos restantes (8.2\%) a los casos mixtos (Figura 1).

\section{Figura 1}

Tipo de delito.

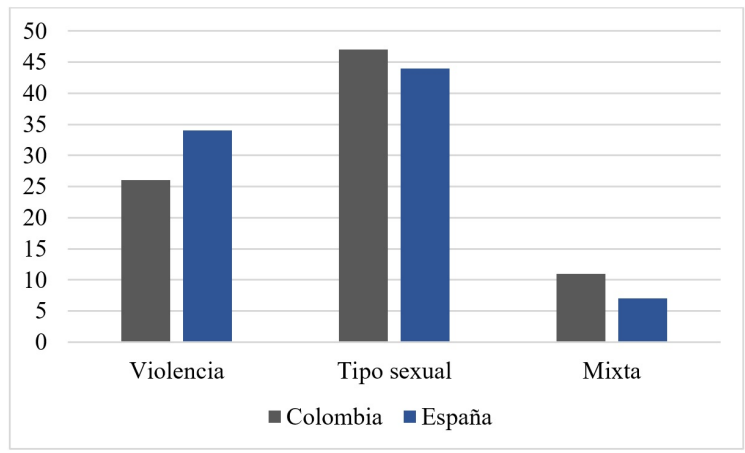

Respecto a la variable dependiente, mención del informe pericial, dentro de los 157 procedimientos revisados se advierte que de las 72 (100\%) sentencias analizadas en Colombia, nueve contienen informes o peritajes psicológicos, lo que corresponde a nueve de los casos $(12.5 \%)$ y 63 de los casos (87.5\%) no contienen informes periciales.

De las sentencias analizadas $(100 \%$ de la población analizada) que poseen pericial psicológica, siete $(77.7 \%)$ contienen entrevista psicológica a la víctima y dos de ellas describen entrevista a testigos dentro del informe psicológico, lo que corresponde a un $22.2 \%$. Se observa, además, que se aplican instrumentos psicométricos y proyectivos en el $33.3 \%$ de los casos.

De las 85 sentencias emitidas en la provincia de Santa Cruz de Tenerife todas tienen informe pericial psicológico forense, con entrevista a la víctima, análisis de credibilidad del testimonio basado en criterios (entrevistas en formato de relato libre, CBCA y SVA, efectuadas por psicólogos peritos, con más de 10 años de experiencia) y aplicación de instrumentos psicométricos y/o proyectivos (solo en menores de 6 años). En 37 (43.5\%) de los casos, las sentencias no mencionan el informe pericial, pero en 48 (56.5\%), sí se hace mención (Figura 2). 
Figura 2

Mención del informe pericial.

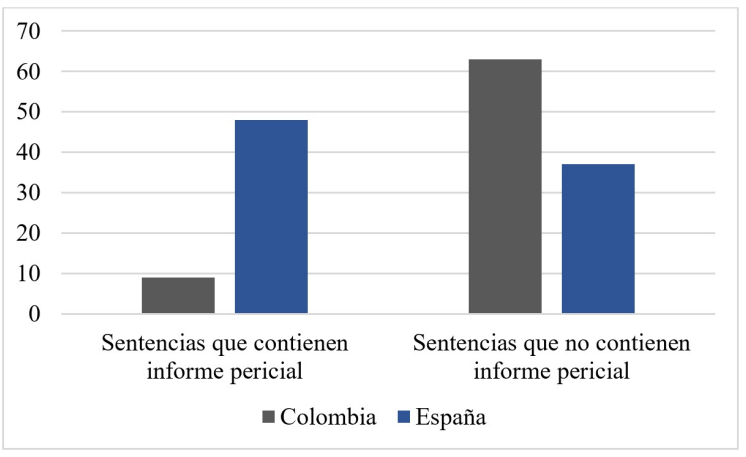

Sin embargo, correlacionando la variable mención de la pericial en la sentencia (variable dependiente) con la variable celebración del juicio (variable independiente) -para ratificación del informe en vista oral: cuando no hay acuerdo entre las partes previo al juicio (75.3\% de la muestra)-, los resultados son tremendamente concluyentes a favor del informe pericial psicológico forense. En el $91.7 \%$ de los casos, en los que se menciona el informe en la sentencia, el juicio se celebra $\left(\chi^{2}(1, n=85)=\right.$ 15.89; $p<0.001 ; \phi=0.432$ ) (Tabla 1$)$.

Tabla 1

Estimaciones de chi cuadrado y Phi

\begin{tabular}{lrr}
\hline & \multicolumn{2}{c}{ Nivel de } \\
& Valor & significancia $(p)$ \\
\hline Chi-cuadrado & 15.89 & 0.000 \\
Phi & 0.43 & 0.000 \\
$\mathrm{~N}$ & 85 & \\
\hline & $* p<0.001$
\end{tabular}

Dicho de otro modo, el estudio realizado en España determinó que cuando se correlaciona la mención del informe pericial, tanto con los juicios celebrados como en los no celebrados, se encuentra que en aquellos celebrados se hace la mención de la pericial en el 91.7\%. Este dato se convierte en prueba inequívoca de la trascendencia para el juzgador.

En cuanto a los juicios no celebrados en sala (aunque la judicatura sí emite sentencia, tras acuerdo por conformidad de las partes, sin necesidad de juicio oral), se encuentra que en el $45.9 \%$ de los casos se menciona la pericial y en el $8.3 \%$ no se menciona el informe psicológico (Figura 3).

\section{Figura 3}

Celebración del juicio.

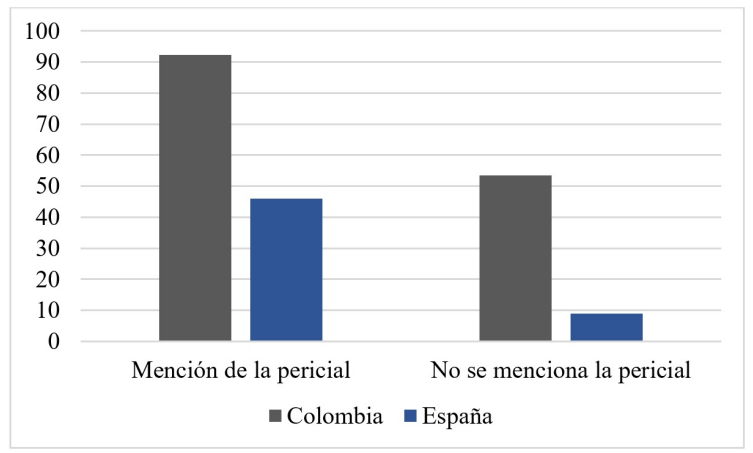

Otra de las variables analizadas en este estudio es la influencia de la pericial (en todos los juicios, celebrados o no) en la toma de decisiones judiciales (es decir, si el fallo va en la línea de las conclusiones del informe o no, con independencia de que haya mención de la pericia en el veredicto). Se observa que en las sentencias analizadas en Santa Cruz de Tenerife, en 25 $(29.4 \%)$ de los casos no se registra influencia de la pericial; mientras que en 59 (70.6\%) de las sentencias sí se halla influencia de los informes periciales en las decisiones judiciales.

En Colombia, $88.8 \%$ de las sentencias analizadas tiene influencia el contenido del informe o peritaje psicológico en la decisión judicial; en el $11.1 \%$ no se advierte tal influencia. En el 88.8\% de las sentencias con esta categoría, los magistrados refieren que las conclusiones del informe psicológico son la base fundamental de su decisión. En el 55.5\% de las sentencias, los magistrados otorgan credibilidad a los asertos de las quejosas, y dicha calificación la hacen con base en los conceptos de los especialistas en psiquiatría y psicología (Figura 4). 


\section{Figura 4}

Influencia de la pericial en la toma de decisión.

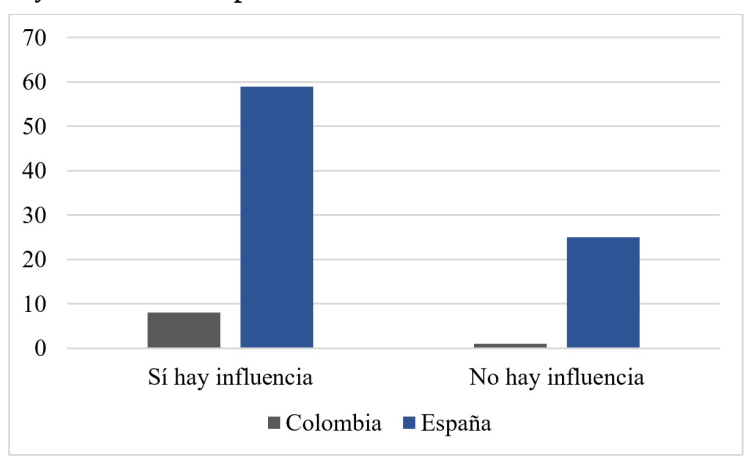

El efecto de las huellas, de memoria y psíquica, dentro de las sentencias revisadas en España arroja resultados dispares. La correlación de cada una de ellas con la alusión del juez (o magistrado) a la prueba pericial psicológica forense en la sentencia, es heterogénea. Con respecto a la huella de memoria (es decir, cuando el relato de las/los evaluados se califica como creíble), se menciona la pericial en el $57.4 \%$ de los veredictos, pero cuando se tiene en cuenta la huella psicológica (entendida como huella primaria: trastorno por estrés postraumático o trastorno adaptativo), se alude a la pericia en el $5.9 \%$ de las sentencias.

En Colombia, se encontró que cuando se correlaciona la VD y la huella de memoria, aquella se nombra en el $66.6 \%$ de las sentencias. Además, en un $55.5 \%$ se identifican sentencias en las que se menciona la VD en relación con la psíquica (Figura 5).

\section{Figura 5}

Huella (psíquica y memoria) vs. mención.

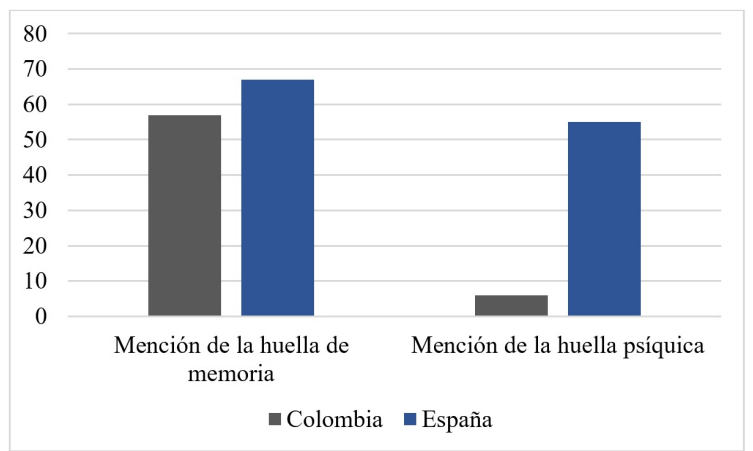

\section{Conclusiones y discusión}

Antes de la discusión de las implicaciones de los resultados, es necesario considerar las limitaciones que se deben tener en cuenta a la hora de generalizar y considerar los resultados. En primer lugar, las muestras, aunque son absolutamente representativas de las poblaciones de abuso sexual infantil a las que representan $(100 \%$ en los departamentos colombianos de Cauca, Nariño y Caldas, y un tamaño superior de muestreo probabilístico simple sin reemplazo en la muestra de Santa Cruz de Tenerife), podrían ser más prolíficas, fiables y válidas si el abanico de casos fuera mayor y más prolongado temporalmente. En segundo lugar, y como consecuencia de lo anterior, se asumió equiparación intercultural y no se tienen garantías plenas de la fidelidad: es posible que las muestras no sean totalmente similares por posibles diferencias culturales no atendidas ni advertidas. En tercer lugar, pese a que la casuística (abuso sexual infantil) es universal, la generalización puede estar comprometida; máxime cuando la literatura científica revisada no reporta estudios similares de transculturalidad, más allá del estudio de Collings et al. (2009). Aunque esta limitación se convierte a la vez en una virtud, porque resulta pionera la revisión efectuada, no se han encontrado investigaciones similares publicadas ni en lengua hispana ni anglosajona, como tampoco la literatura científica revisada aporta datos transculturales entre España y Colombia referidos a delitos sexuales.

Con estas precisiones muy presentes, se puden extraer conclusiones que dan respuesta a las hipótesis establecidas en este estudio, a saber: iqué uso hace el juzgador de la prueba pericial sobre delitos sexuales infantiles (o discapacitados) en España y en Colombia?, ipara el buen funcionamiento de la Justicia, el derecho puede resolver las situaciones de abuso sexual solo con códigos y leyes, y sin la aportación de la psicología forense? En consecuencia, se concluye que el presente estudio verifica que los juzgadores conceden relevancia a la prueba técnica (pericial) realizada por el poseedor de 
conocimiento científico (pericia) en el contexto judicial de abuso sexual infantil. Estudios anteriores también indican que desde el ámbito del derecho interesa no solo la producción de la prueba a partir de la contribución de la psicología, sino el manejo de los casos de índole sexual a través de los profesionales de la psicología forense (Granjeiro \& Costa, 2010). Del mismo modo y de acuerdo con Tejero y González (2009), la psicología forense es un área de especialización en la que los peritos asesoran a los jueces. Así, en torno al 90\% de las sentencias analizadas en la que se celebra vista oral se hace alusión explícita al informe pericial (variable dependiente de este estudio), porcentaje que se repite tanto en las sentencias redactadas en España como en Colombia. Los datos obtenidos están en consonancia con los hallados por López y Pueyo (2007), esto es, en torno al 88\%; a estos datos se llega con independencia del código de procedimiento penal reinante, con reforma legislativa o sin ella, en un país o en otro. De manera que la prueba pericial psicológica forense se revela como un instrumento válido, productivo y fiable al servicio de los administradores de Justicia. Los resultados mejoran ligeramente los obtenidos en 2013 por Moreno et al., que hallaron en un estudio similar que el $73 \%$ de las veces en las que se cita la pericial psicológica en la sentencia judicial, esta va aparejada con abuso sexual. En la misma línea, Rudas et al. (2016) reportan que en un $80 \%$ de los casos. el juez tomó su decisión de manera concordante con los resultados del informe psicológico forense.

Por otro lado, la distancia física y sobre todo las peculiaridades costumbristas, razón del derecho consuetudinario, entre Colombia y España no conllevan a diferencias significativas en los resultados. Ni siquiera las divergencias metodológicas y procedimentales en la elaboración de los informes, se traducen en impedimentos para hallar similitudes; es más, se convierten en un puente de unión entre las pericias de las dos orillas y tienen influencia en la decisión judicial en la administración de Justicia.

Pero la exaltación de las semejanzas puede ser excesiva. Se están generalizando conclusiones a todos los ámbitos del derecho y de la psicología forense cuando en realidad únicamente hemos desarrollado una investigación, con las limitaciones advertidas, ceñida al derecho penal y encorsetada en el perímetro de la psicología del testimonio infantil. No solo se han pasado por alto otros campos de trabajo del psicólogo forense en terrero de lo penal (p. ej., evaluación de cognición y volición), sino que no se referencias pericias efectuadas al servicio del derecho civil, laboral, etc. No obstante, lo que no se puede negar es que este trabajo supone un compromiso inédito de coparticipación entre profesionales de dos ámbitos, tan distintos a veces, pero tan cerca en otras ocasiones. En este caso, la proximidad entre psicología y derecho va más allá de la confluencia entre la toga y la bata, negra la primera y blanca la segunda, ambas, valoran aspectos de la conducta humana y supuestos legales. Si definimos la psicología forense como la ciencia que aplica todas las ramas y saberes de la psicología a las preguntas de la justicia, actuando en el foro y mejorando el ejercicio del derecho, este artículo se esfuerza en responder cuánto influye la psicología y en qué medida acude al auxilio del derecho, aunque solo se aborda una pequeña gama de los diversos campos de influencia de la psicología.

Sin embargo, como se ha expuesto, la carencia más sustancial del trabajo atañe a la muestra. Esta deficiencia, en Colombia, es la consecuencia de un sistema judicial con escasa influencia del informe pericial hasta el periodo de recogida de la muestra (aunque no deja de ser significativo que se incluya al 100\% de la población). No menos importante es subrayar que la muestra proviene de tribunales de segunda instancia y de sentencias dimanadas de informes periciales psicológicos de oficio. Es decir, como consecuencia de mandatos judiciales (o de la Fiscalía) de las administraciones de Justicia española o colombiana; no se sabe qué ocurre con las sentencias de primera instancia ni con los informes periciales psicológicos forenses de parte. En cuanto a la muestra española, las sentencias provienen de primera y segunda instancias, y representa una sola de las 17 comunidades 
autónomas del Estado. Estas limitaciones deben ser el punto de partida de investigaciones futuras.

Por último, el valor de este trabajo es introductorio y exploratorio, pero une a dos realidades tan similares, como a la vez, diferentes, a través de los diferentes tonos y colores de la pericial psicológica, y posee el valor añadido de la novedad. Es inédito y subraya la importancia de la psicología como ciencia auxiliar del derecho. Además, deja la puerta abierta a líneas de investigación futuras en la aclaración de los hechos; sobre todo, estudios dirigidos a la protección de los menores y sus familias en un área donde la falta de evidencia, en forma de secuelas físicas, obliga a integrar diferentes fuentes de información. Esta realidad, de no revictimizar, se repite en Cauca, Caldas, Nariño y Santa Cruz de Tenerife, en Colombia o España. De modo que, a partir de ahora se abre una línea de investigación futura con repercusiones y utilidades que trascienden el carácter exploratorio de estos resultados y la pretensión inicial del estudio.

\section{Referencias}

Aliste Santos, T. J. (2011). La motivación de las resoluciones judiciales. Madrid: Marcial Pons.

Centro Reina Sofía para el Estudio de la Violencia. (2004). Abuso sexual infantil, Credibilidad del testimonio. Estudio de 100 casos. Valencia, ES: Diseñarte-Goaprint, S. L.

Collings, S. J., Lindblom, L., Madu, S. N., \& Park, M. S. (2009). The cross-cultural validity of the Child Sexual Abuse Myth Scale: A preliminary investigation. Journal of Psychology in Africa, 19(1), 9-17. http://dx. doi.org/10.1080/14330237.2009.10820253

Colombia, Código de Procedimiento Penal [CPP]. Ley 600 de 2000. Julio 24 de 2000.

Colombia, Código de Procedimiento Penal [CPP]. Ley 906 de 2004. Agosto 31 de 2004.

España, Constitución Española. Don Juan Carlos I. Rey de España. Octubre 31 de 1978.
España, Tribunal Supremo. Sentencia 310 del 6 de marzo de 1995. RJ 1995\1811.

Fundación Ayuda a Niños y Adolescentes en Riesgo. (2012). Día Internacional de la Lucha contra el Maltrato Infantil (Informe de Violencia contra la Infancia). Madrid: Autor. Recuperado de http://www.anar.org/wp-content/upload s/2013/04/Informe-ANAR-2012-BAJA.pdf

Granjeiro, I., \& Costa, L. F. (2010). A interdisciplinaridade entre direito e psicologia no conflito familiar violento. Revista de Informação Legislativa, 47(185), 195-209. Recuperado de http://www2.senado.leg.br/bdsf/bitstrea $\mathrm{m} /$ handle/id/198667/000881218.pdf?seque nce $=1$

Hernández, G. (2008). Fundamentos de derecho para psicólogos. En A. Tapias \& C. Gutiérrez de Piñeres (Coords.), Psicología jurídica: perspectiva latinoamericana (p. 83). Bogotá: Edición independiente.

López, S., \& Pueyo, A. (2007). El efecto del peritaje psicológico en los juicios contra la libertad sexual de menores. Colección Psicología y Ley (N. ${ }^{\circ}$ 3, pp. 27-32). Santiago de Compostela: Sociedad Española de Psicología Jurídica y Forense.

Moreno, B., Correa, J., \& Pérez, I. (2013). Reflexiones sobre psicología jurídica desde el Teide. En Nuevos aportes de la psicología jurídica (pp. 151-157). Madrid: EOS.

Mrazek, P. J. (1983). Sexual Abuse of Children. En B. B. Lahey \& A. E. Kazdin (Eds.), Advances in Clinical Child Psychology (Vol 6., pp. 199-215). Boston, MA: Springer. https: //dx.doi.org/10.1007/978-1-4613-9814-1_6

Pereda, N. (2016). ¿Uno de cada cinco?: victimización sexual infantil en España. Papeles del Psicólogo, 37(2), 126-133. Recuperado de http://www.papelesdelpsico logo.es/pdf/2697.pdf

Pompeyo, M., \& López, R. (2014). La intervención del perito en el sistema penal acusatorio. Revista Digital de la Reforma Penal, Nova Iustitia, 6, 1-204. Recuperado de https://docslide.net/documents/6-revist a-nova-iustitia-febrero-2014.html 
Raskin, D. C., \& Steller, M. (1989). Assesing credibility of allegations of child sexual abuse: Poligraph examinations and statement analysis. En H. Wegener, F. Lösel \& J. Haisch (Eds.), Criminal behavior and the justice system (pp. 290-302). Nueva York: Springer-Verlag.

Rodríguez, H. (2010). Evaluación médicolegal del abuso sexual infantil: revisión y actualización. Cuadernos de Medicina Forense, 16(1-2), 99-108. Recuperado de http://scielo.isciii.es/pdf/cmf/v16n1-2/re vision10.pdf

Rudas, M. M., Baena, S., \& Pérez, I. (2016). Peritajes psicológicos forenses en decisiones judiciales de primera instancia en delitos sexuales. Revista de Derecho, 46, 200-2024. http://dx.doi.org/10.14482/dere.46.8816

Simoes, M. (2001). Informes psicológicos en contexto forense. En F. Jiménez (Coord.), Evaluación psicológica forense: ámbitos delictivos, laboral y elaboración de informes (pp. 157-183). Salamanca, ES: Amarú Ediciones.

Soria, M., \& Saiz, D. (2006). Psicología criminal. Madrid: Prentice Hall.

Stoltenborgh, M., Van Ljzendoorm, M. H., Eurser, E. M., \& Bakermans-Kranenburg, M. J. (2011). A global perspective on child sexual abuse: Metaanalysis of prevalence around the world. Child Maltreatment, 16(2), 79-101. http://dx.doi.org/10.1177/1 077559511403920

Tapias, A. (2011). Técnicas psicológicas forenses en caso de retractación de la víctima de delito sexual menor de edad. Revista Iusta, 35, 53-79. Recuperado de https://doctrina.vlex.com.co/vid/forense s-va-ctima-delito-sexual-menor-43619997 4

Tejero, R., \& González, D. (2009). Informe pericial psicológico. Abuso sexual infantil. Revista Argentina de Clínica Psicológica, 18(1), 77-88. Recuperado de http://www.cienciared.com.ar/ra/usr/35/ 762/racp_xviii_1_pp77_88.pdf

\section{Notas}

* Artículo de investigación. La presente investigación hace parte del proyecto "Importancia de los peritajes psicológicos forenses en el ámbito del derecho, estudios nacionales y comparados" de la Universidad de la Costa CUC (Colombia). 